\title{
A New Definition for Generalized First Derivative of Nonsmooth Functions
}

\author{
Ali Vahidian Kamyad, Mohammad Hadi Noori Skandari, Hamid Reza Erfanian \\ Department of Applied Mathematics, School of Mathematical Sciences, \\ Ferdowsi University of Mashhad, Mashhad, Iran \\ E-mail: \{avkamyad, hadinoori344,erfanianhamidreza\}@yahoo.com \\ Received July 26, 2011; revised August 29, 2011; accepted September 6, 2011
}

\begin{abstract}
In this paper, we define a functional optimization problem corresponding to smooth functions which its optimal solution is first derivative of these functions in a domain. These functional optimization problems are applied for non-smooth functions which by solving these problems we obtain a kind of generalized first derivatives. For this purpose, a linear programming problem corresponding functional optimization problem is obtained which their optimal solutions give the approximate generalized first derivative. We show the efficiency of our approach by obtaining derivative and generalized derivative of some smooth and nonsmooth functions respectively in some illustrative examples.
\end{abstract}

Keywords: Generalized Derivative, Smooth and Nonsmooth Functions, Fourier analysis, Linear Programming, Functional Optimization

\section{Introduction}

The generalized derivative has played an increasingly important role in several areas of application, notably in optimization, calculus of variations, differential equations, Mechanics, and control theory (see [1-3]). Some of known generalized derivatives are the Clarke's Generalized derivative [1], the Mordukhovich's Coderivatives [4-8], Ioffe's Prederivatives [9-13], the Gowda and Ravindran H-Differentials [14], the Clarck-Rockafellar Subdifferential [15], the Michel-Penot Subdifferential [16], the Treiman's Linear Generalized Gradients [17], and the Demyanov-Rubinov Quasidifferentials [2]. In these mention works, for introducing generalized derivative of non-smooth function $f($.$) on interval [a, b]$, some restrictions and results there exist, for examples

1) The function $f($.$) must be locally Lipschitz or$ convex.

2) We must know that the function $f($.$) is non-dif-$ ferentiable at a fixed number $x \in[a, b]$.

3) The obtained generalized derivative of $f($.$) at$ $x \in[a, b]$ is a set which may be empty or including several members.

4) The directional derivative is used to introduce generalized derivative.

5) The concepts limsup and liminf are applied to obtain the generalized derivative in which calculation of these is usually hard and complicated.

However, in this paper, we propose a new definition for generalized first derivative (GFD) which is very useful for practical applications and hasn't above restrictions and complications. We introduce an especially functional optimization problem for obtaining the GFD of nonsmooth functions. This functional optimization problem is approximated with the corresponding linear programming problem that we can solve it by linear programming methods such as simplex method.

The structure of this paper is as follows. In Section 2, we define the GFD of non-smooth functions which is based on functional optimization. In addition, we introduce the linear programming problem to obtain the approximate GFD of non-smooth functions. In Section 3, we use our approach for smooth and nonsmooth functions in some examples. Conclusions of this paper will be stated in Section 4.

\section{GFD of Nonsmooth Functions}

In this section, we are going to introduce a functional optimization problem that its optimal solution is the derivative of smooth function on interval $[0,1]$. For solving this problem, we introduce a linear programming 
problem. So, by solving this problem for nonsmooth functions, we obtain an approximate derivative for these functions on interval $[0,1]$ which is GFD. Firstly, we state the following Lemma which $C[0,1]$ is space of continuous functions on $[0,1]$ :

Lemma 2.1: Let $h(.) \in C[0,1]$ and $\int_{0}^{1} \eta(x) h(x) \mathrm{d} x=0$ for any $\eta(.) \in C[0,1]$. We have $h(x)=0$ for all $x \in[0,1]$.

Proof: Consider $x_{0} \in[0,1]$ such that $h\left(x_{0}\right) \neq 0$. Without loss of generality, suppose $h\left(x_{0}\right)>0$. Since $h(.) \in C[0,1]$, then there is a neighborhood $N\left(x_{0}, \delta\right)$, $\delta>0$, of $x_{0}$ such that $h(x)>0$ for all $x \in N\left(x_{0}, \delta\right)$. We consider the functin $\eta_{0}(.) \in C[0,1]$ such that $\eta_{0}($. is zero on $[0,1]-N\left(x_{0}, \delta\right)$ and positive on $N\left(x_{0}, \delta\right)$. Thus we have:

$$
0<\int_{N\left(x_{0}, \delta\right)} \eta_{0}(x) h(x) \mathrm{d} x=\int_{0}^{1} \eta_{0}(x) h(x) \mathrm{d} x
$$

so $\int_{0}^{1} h(x) \eta_{0}(x) d x>0$ which is a contradiction. Then $h(x)=0$ for all $x \in[0,1]$.

Now we state the following theorem and prove it by Lemma 2.1 which $C^{1}[0,1]$ is the space of differentiable functions with the continuous derivative on $[0,1]$.

Theorem 2.2: Let $f(),. g(.) \in C[0,1]$ and $\int_{0}^{1}\left(v^{\prime}(x) f(x)+v(x) g(x)\right) \mathrm{d} x=0$ for any $v(.) \in C^{1}[0,1]$ where $v(0)=v(1)=0$. Then we have $f(.) \in C^{1}[0,1]$ and $f^{\prime}()=.g($.$) .$

Proof: We use integration by parts rule and conditions $v(0)=v(1)=0$ :

$$
\begin{aligned}
& \int_{0}^{1} v(x) g(x) \mathrm{d} x=[v(x) G(x)]_{0}^{1}-\int_{0}^{1} v^{\prime}(x) G(x) \mathrm{d} x \\
& =[v(1) G(1)-v(0) G(0)]-\int_{0}^{1} v^{\prime}(x) G(x) \mathrm{d} x \\
& =-\int_{0}^{1} v^{\prime}(x) G(x) \mathrm{d} x .
\end{aligned}
$$

where $G(x)=\int_{0}^{x} g(t) \mathrm{d} t$ for each $x \in[0,1]$. Since $\int_{0}^{1}\left(v^{\prime}(x) f(x)+v(x) g(x)\right)=0$, so by relation (1),

$$
\int_{0}^{1} v^{\prime}(x) G(x) \mathrm{d} x=\int_{0}^{1} v^{\prime}(x) f(x)
$$

or

$$
\int_{0}^{1} v^{\prime}(x)(G(x)-f(x)) \mathrm{d} x=0 .
$$

Put $h(x)=G(x)-f(x)$ for each $x \in[0,1]$. By Lemma 2.1, since $v^{\prime}(.) \in C[0,1]$ we have $h(x)=0$ for all $x \in[0,1]$, it means that $G()=.f($.$) . Thus$

$$
f(x)=\int_{0}^{x} g(t) \mathrm{d} t . \text { So } f(.) \in C[0,1] \text { and } f^{\prime}(.)=g(.) \text {. }
$$

let

$$
V=\left\{v_{k}(.): v_{k}(x)=\sin k \pi x: x \in[0,1], k=1,2, \cdots\right\} .
$$

Then $v_{k}(0)=v_{k}(1)=0$ for all $v_{k}(.) \in V$. We can extend every continuous function $v(.) \in C[0,1]$ which satisfies $v(0)=0$ as an odd function on $[-1,1]$. Thus, there is a Sinus expansion for this function on $[-1,1]$. Now we have the following theorem:

Theorem 2.3: Let $f(),. g(.) \in C[0,1]$ and $\int_{0}^{1}\left(v_{k}^{\prime}(x) f(x)+v_{k}(x) g(x)\right) \mathrm{d} x=0$ where $v_{k}(.) \in V$. Then we have $f(.) \in C^{1}[0,1]$ and $f^{\prime}()=.g($.$) .$

Proof: Let $\hat{V}=\left\{v(.) \in C^{1}[0,1]: v(0)=v(1)=0\right\}$ and $v(.) \in \hat{V}$. Since set $V$ is a total set for space $\widehat{V}$, There are real coefficients $c_{1}, c_{2}, \cdots$ such that $v(x)=\sum_{k=1}^{\infty} c_{k} v_{k}(x)$ for any $x \in[0,1]$ where $v_{k}(.) \in V$. Thus, if

$$
\int_{0}^{1}\left(v_{k}^{\prime}(x) f(x)+v_{k}(x) g(x)\right) \mathrm{d} x=0, \quad k=1,2, \cdots
$$

then

$$
\sum_{k=1}^{\infty} c_{k}\left(\int_{0}^{1}\left(v_{k}^{\prime}(x) f(x)+v_{k}(x) g(x)\right) \mathrm{d} x\right)=0
$$

We know the series $\sum_{k=1}^{\infty} c_{k} v_{k}($.$) is uniformly conver-$ gent to the function $v($.$) . So by relation (2)$

$$
\int_{0}^{1}\left(\sum_{k=1}^{\infty} C_{k} v_{k}^{\prime}(x) f(x)+\sum_{k=1}^{\infty} c_{k} v_{k}(x) g(x)\right) \mathrm{d} x=0
$$

Thus, by (3)

$$
\int_{0}^{1}\left(v^{\prime}(x) f(x)+v(x) g(x) \mathrm{d} x\right)=0 .
$$

where $v^{\prime}(x)=\sum_{k=1}^{\infty} c_{k} v_{k}^{\prime}(x)$. Thus for any $v(.) \in \hat{V}$ the relation (4) and conditions of theorem 2.2 hold. Then we have $f(.) \in C^{1}[0,1]$ and $f^{\prime}()=.g($.$) . \square$

Now we state the following theorem and in next step use it.

Theorem 2.4: Let $\varepsilon>0$ is given small number, $f(.) \in C^{1}[0,1]$ and $m \in \mathbb{N}$. Then there exist $\delta>0$ such that for all $s_{i} \in\left(\frac{i-1}{m}, \frac{i}{m}\right), \quad i=1,2, \cdots, m$

$$
\int_{s_{i}-\delta}^{s_{i}+\delta}\left|f(x)-f\left(s_{i}\right)-\left(x-s_{i}\right) f^{\prime}\left(s_{i}\right)\right| \mathrm{d} x \leq \varepsilon \delta^{2}
$$

Proof: Since $f^{\prime}\left(s_{i}\right)=\lim _{x \rightarrow s_{i}} \frac{f(x)-f\left(s_{i}\right)}{x-s_{i}}, i=1,2, \cdots, m$, so there exist $\delta_{s_{i}}>0$ such that for all 


$$
\begin{aligned}
& x \in\left[s_{i}-\delta_{i}, s_{i}+\delta_{i}\right] \\
& \quad\left|\frac{f(x)-f\left(s_{i}\right)}{x-s_{i}}-f^{\prime}\left(s_{i}\right)\right| \leq \frac{\varepsilon}{2}, i=1,2, \cdots, m
\end{aligned}
$$

Suppose that $\delta=\min \left\{\delta_{s_{i}}, i=1,2, \cdots, m\right\}$. So for all $s_{i} \in\left(\frac{i-1}{m}, \frac{i}{m}\right)$ and $x \in\left[s_{i}-\delta, s_{i}+\delta\right], i=1,2, \cdots, m$ inequality (6) is satisfied. Thus $\left[s_{i}-\delta, s_{i}+\delta\right] \subseteq\left[s_{i}-\delta_{s_{i}}, s_{i}+\delta_{s_{i}}\right]$ and by inequality (6) we obtain for all $i=1,2, \cdots, m$

$$
\left|f(x)-f\left(s_{i}\right)-\left(x-s_{i}\right) f^{\prime}\left(s_{i}\right)\right| \leq \frac{\varepsilon}{2}\left|x-s_{i}\right| \leq \frac{\varepsilon}{2} \delta,
$$

Thus by integrating both sides of inequality (7) on interval $\left[s_{i}-\delta, s_{i}+\delta\right], i=1,2, \cdots, m$ we can obtain inequality (5).

Now consider the continuous functions $f($.$) and$ $g($.$) such that \int_{0}^{1}\left(v_{k}^{\prime}(x) f(x)+v_{k}(x) g(x)\right) \mathrm{d} x=0$ for any $v_{k}(.) \in V$. We have:

$$
\int_{0}^{1} v_{k}(x) g(x) \mathrm{d} x=\lambda_{k}, v_{k}(.) \in V, k=1,2,3, \cdots
$$

where

$$
\lambda_{k}=-\int_{0}^{1} v_{k}^{\prime}(x) f(x) \mathrm{d} x, k=1,2,3, \cdots
$$

Let $\varepsilon>0$ and $\delta>0$ are two sufficiently small given number and $m \in \mathbb{N}$. For given continuous function $f(x)$ on $[0,1]$, we define the following functional optimization problem:

minimize

$$
J(g(.))=\sum_{k=1}^{\infty}\left|\int_{0}^{1} v_{k}(x) g(x) \mathrm{d} x-\lambda_{k}\right|
$$

subject to

$$
\begin{aligned}
& \int_{s_{i}-\delta}^{s_{i}+\delta}\left|f(x)-f\left(s_{i}\right)-\left(x-s_{i}\right) g\left(s_{i}\right)\right| \mathrm{d} x \leq \varepsilon \delta, \\
& g(.) \in C[0,1], s_{i} \in\left(\frac{i-1}{m}, \frac{i}{m}\right), i=1,2, \cdots, m
\end{aligned}
$$

where $v_{k}(.) \in V$ for all $k=1,2,3, \cdots$.

Theorem 2.5: Let $f(.) \in C^{1}[0,1]$ and $g^{*}(.) \in C[0,1]$ be the optimal solution of the functional optimization problem (10)-(11). Then, $f^{\prime}()=.g^{*}($.$) .$

Proof: It is trivial $\sum_{k=1}^{\infty}\left|\int_{0}^{1} v_{k}(x) g(x) \mathrm{d} x-\lambda_{k}\right| \geq 0$ for all $g(.) \in C[0,1]$. By theorem 2.3, we have $\left|\int_{0}^{1} v_{k}(x) g(x) \mathrm{d} x-\lambda_{k}\right|=0$ for all $v_{k}(.) \in V$ where $g()=.f^{\prime}($.$) on [0,1]$. So

$$
\sum_{k=1}^{\infty}\left|\int_{0}^{1} v_{k}(x) f^{\prime}(x) \mathrm{d} x-\lambda_{k}\right|=0,
$$

hence

$$
0=\sum_{k=1}^{\infty}\left|\int_{0}^{1} v(x) f^{\prime}(x) \mathrm{d} x-\lambda_{k}\right| \leq \sum_{k=1}^{\infty}\left|\int_{0}^{1} v(x) g(x) \mathrm{d} x-\lambda_{k}\right|
$$

On the other hand $f^{\prime}(.) \in C[0,1]$ and by theorem 2.4 function $f^{\prime}($.$) satisfies in constraints of problem (10)-$ (11). Thus, $f^{\prime}($.$) is optimal solution of functional op-$ timization (10)-(11). $\square$

Thus, the GFD of non-smooth functions may be defined as follows:

Definition 2.6: Let $f($.$) be a continuous nonsmooth$ function on the interval $[0,1]$ and $g^{*}($.$) be the opti-$ mal solution of the functional optimization problem (10)(11). We denote the GFD of $f($.$) by G F_{d} f($.$) and$ define as $G F_{d} f()=.g^{*}($.$) .$

Remark 2.7: Note that if $f($.$) be a smooth function$ then the $G F_{d} f($.$) in definition 2.6$ is $f^{\prime}($.$) . Further, if$ $f($.$) be a nonsmooth function then GFD of f($.$) is an$ approximation for first derivative of function $f($.$) .$

However, the functional optimization problem (10)(11) is an infinite dimensional problem. Thus we may convert this problem to the corresponding finite dimensional problem. We can extend any function $g(.) \in C[0,1]$ on interval $[-1,1]$ as Fourier series

$$
g(x)=\frac{a_{0}}{2}+\sum_{n=1}^{\infty}\left(a_{k} \cos (k \pi x)+b_{k} \cos (k \pi x)\right)
$$

where coefficients $a_{k}$ and $b_{k}$ for $k=1,2, \cdots$ satisfying in the following relations:

$$
\begin{aligned}
& a_{k}=\int_{-1}^{1} \cos (k \pi x) g(x) \mathrm{d} x, \\
& b_{k}=\int_{-1}^{1} \sin (k \pi x) g(x) \mathrm{d} x,
\end{aligned}
$$

On the other hand, by Fourier analysis (see [18]) we have $\lim _{k \rightarrow \infty} a_{k}=0$ and $\lim _{k \rightarrow \infty} b_{k}=0$. Then there exists $N \in \mathbb{N}$ such that for all $k \geq N+1$ we have $a_{k} \approx 0$ and $b_{k} \approx 0$.

Hence, the problem (10)-(11) is approximated as the following finite dimensional problem:

minimize

$$
J(g(.))=\sum_{k=1}^{N}\left|\int_{0}^{1} v_{k}(x) g(x) \mathrm{d} x-\lambda_{k}\right|
$$

subject to

$$
\begin{aligned}
& \int_{s_{i}-\delta}^{s_{i}+\delta}\left|f(x)-f\left(s_{i}\right)-\left(x-s_{i}\right) g\left(s_{i}\right)\right| d x \leq \varepsilon \delta^{2}, \\
& g(.) \in C[0,1], s_{i} \in\left(\frac{i-1}{m}, \frac{i}{m}\right), i=1,2, \cdots, m
\end{aligned}
$$


where $N$ is a given large number. We assume that $g_{i}=g\left(s_{i}\right), \quad f_{i 1}=f\left(s_{i}-\delta\right), \quad f_{i 2}=f\left(s_{i}+\delta\right)$ and $f_{i}=f\left(s_{i}\right)$ for all $i=1,2, \cdots, m$. In addition, we choose the arbitrary points $s_{i} \in\left(\frac{i-1}{m}, \frac{i}{m}\right), i=1,2, \cdots, m$ Вy trapezoidal and midpoint integration rules, problem (13)(14) can be written as the following problem which $g_{1}, g_{2}, \cdots, g_{m}$ are its unknown variables:

$$
\text { minimize } \sum_{k=1}^{N}\left|\delta \sum_{i=1}^{m} v_{k i} g_{i}-\lambda_{k}\right|
$$

subject to

$$
\begin{aligned}
& \left|f_{i 1}-f_{i}+\delta g_{i}\right|+\left|f_{i 2}-f_{i}-\delta g_{i}\right| \leq \varepsilon \delta, \\
& i=1,2, \cdots, m
\end{aligned}
$$

Now, problem (15)-(16) may be converted to the following equivalent linear programming problem (see [19, 20]) which $g_{i}, i=1, \cdots, m, \quad \mu_{k}, \quad k=1,2, \cdots, N$ and $\omega_{i}, z_{i}, u_{i}, \quad v_{i}$ for $i=1,2, \cdots, m$ are unknown variables of the problem:

$$
\text { minimize } \sum_{k=1}^{N} \mu_{k}
$$

subject to

$$
\begin{aligned}
& -\mu_{k}+\delta \sum_{i=0}^{m} v_{k i} g_{i} \leq \lambda_{k}, k=1,2, \cdots, N \\
& -\mu_{k}-\delta \sum_{i=0}^{m} v_{k i} g_{i} \leq-\lambda_{k}, k=1,2, \cdots, N \\
& \left(u_{i}+v_{i}\right)+\left(\omega_{i}+z_{i}\right) \leq \varepsilon \delta, i=1,2, \cdots, m \\
& u_{i}-v_{i}-\delta g_{i}=f_{i 1}-f_{i}, i=1,2, \cdots, m \\
& \omega_{i}-z_{i}+\delta g_{i}=f_{i 2}-f_{i}, i=1,2, \cdots, m \\
& \omega_{i}, z_{i}, u_{i}, v_{i}, \mu_{k} \geq 0, k=1,2, \cdots, N ; i=1,2, \cdots, m .
\end{aligned}
$$

where $\lambda_{k}$ for $k=1,2,3, \cdots, N$ are satisfying in relation (9).

Remark 2.8: By relations (9), we may approximate $\lambda_{k}$ for all $k=1,2,3, \cdots, N$ as follows:

$$
\lambda_{k} \simeq-\delta \sum_{i=1}^{m} v_{k i}^{\prime}\left(x_{i}\right) f\left(x_{i}\right)
$$

Moreover, note that $\varepsilon$ and $\delta$ must be sufficiently small numbers and points $s_{i} \in\left(\frac{i-1}{m}, \frac{i}{m}\right), i=1,2, \cdots, m$ can be chosen as arbitrary numbers.

Remark 2.9: Note that if $g_{i}^{*}, i=1, \cdots, m$ be optimal solutions of problem (17), then we have $G F_{d} f\left(s_{i}\right)=g_{i}^{*}$ for $i=1, \cdots, m$.

In next section, we evaluate the GFD of some smooth and nonsmooth functions using our approach

\section{Numerical Examples}

In this stage, we find the GFD of smooth and nonsmooth functions in several examples using problem (17). Here we assume $\varepsilon=\delta=0.01, \quad N=20, \quad m=99$ and $s_{i}=0.01 i$ for all $i=1, \cdots, 99$. The problem (17) is solved for functions in these examples using simplex method (see [19]) in MATLAB software.

Remark 3.1: Note that functions $f($.$) in these ex-$ amples haven't lipschitz or convexity property. Thus we don't use of pervious approaches and methods for obtaining an approximate derivative of function $f($.$) ,$ while with using our approach, we get it.

Remark 3.2: We attend that in our approach points in $[0,1]$ are selected arbitrarily, and with selection very of these points, we can cover this interval. Thus this is a global approach, while the previous approaches and methods act as locally on a fixed given point in $[0,1]$.

Example 3.1: Consider function $f(x)=\exp \left(-(x-0.5)^{2}\right)$ on interval $(0,1)$ which is a smooth function. The function $f(x)=\exp \left(-(x-0.5)^{2}\right)$ is illustrated in Figure 1. Using problem (17), we obtain the GFD of this function which is shown in Figure 2.

Example 3.2: Consider function $f(x)=|| 2 x-1|-0.5|$ on interval $(0,1)$ which is a non-differentiable function in point $x_{i}=0.25 i$ for $i=1,2,3$. The function $f(x)=|| 2 x-1|-0.5|$ is illustrated in Figure 3. We obtain the GFD of function $f($.$) by solving problem (17)$ which is shown in Figure 4.

Example 3.3: Consider function $f(x)=|\cos (5 \pi x)|$ on interval $(0,1)$ which is a non-differentiable function in point $x_{i}=\frac{2 i-1}{10}$ for any $i=1,2, \cdots, 5$. The function $f(x)=|\cos (5 \pi x)|$ and the GFD of this function are shown in Figures 5 and 6 respectively.

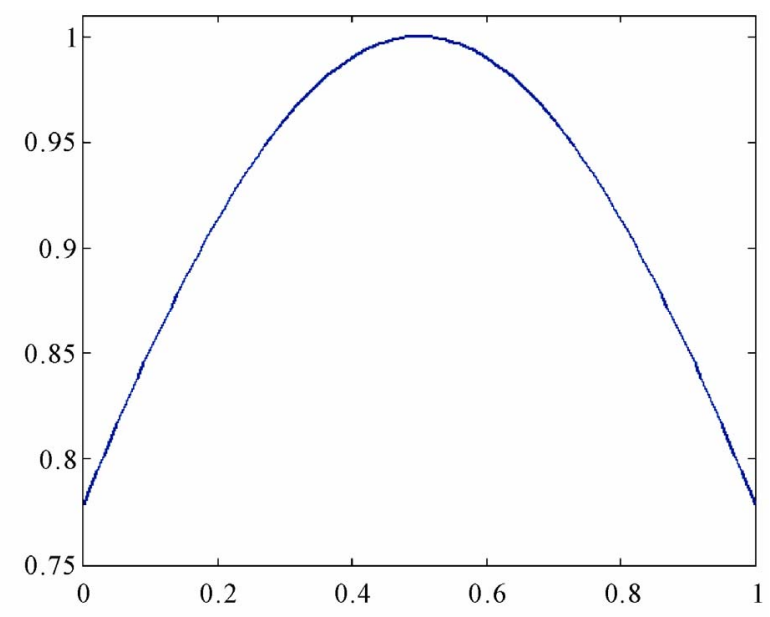

Figure 1. Graph of $f(x)=\exp \left(-(x-0.5)^{2}\right)$. 


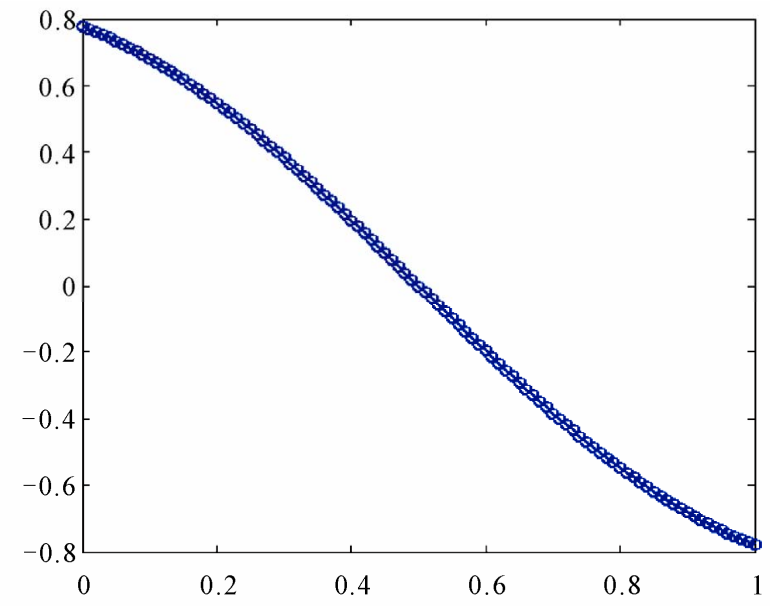

Figure 2. Graph of $G F_{d} f($.$) for Example 3.1.$

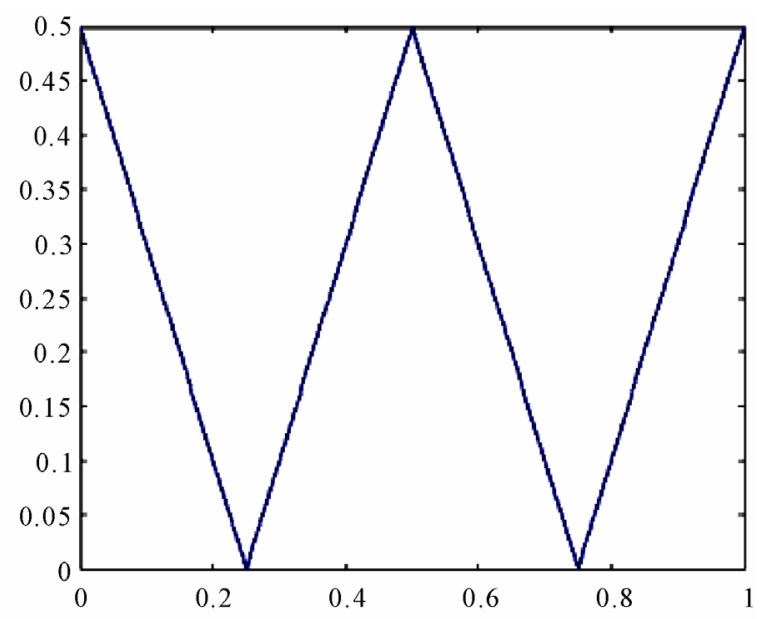

Figure 3. Graph of $f(x)=|| 2 x-1|-0.5|$.

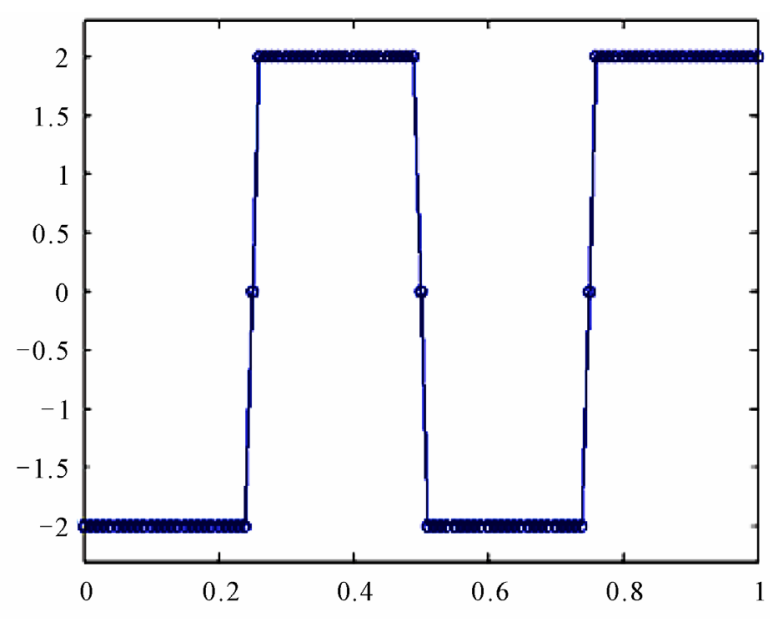

Figure 4. Graph of $G F_{d} f($.$) for Example 3.2.$

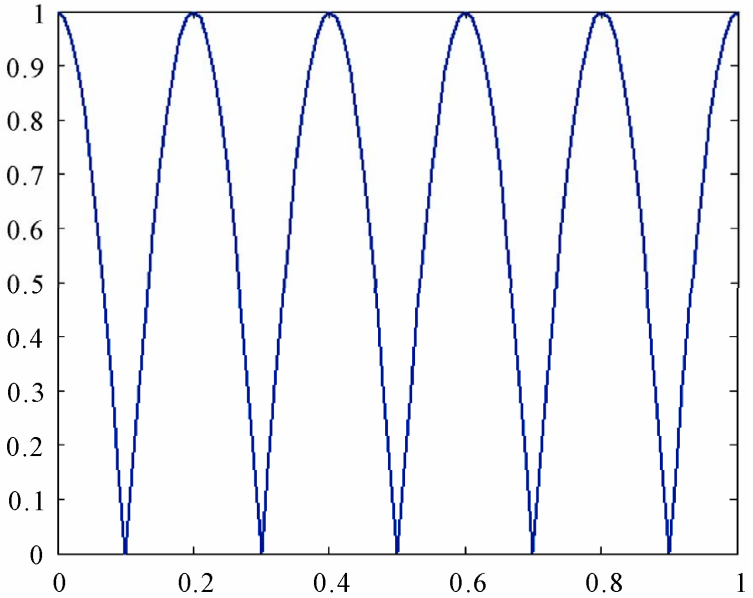

Figure 5. Graph of $f(x)=|\cos (5 \pi x)|$.

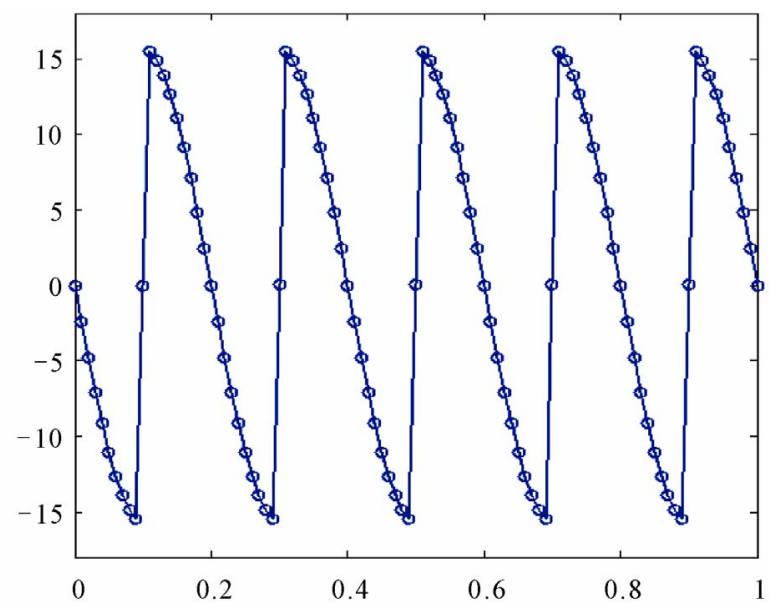

Figure 6. Graph of $G F_{d} f($.$) for Example 3.3.$

\section{Conclusions}

In this paper, we defined a new generalized first derivative (GFD) for non-smooth functions as optimal solution of a functional optimization on the interval $[0,1]$. We approximated this functional optimization problem by a linear programming problem. The definition of GFD in this paper has the following properties and advantages:

1) Here, the obtained GFD For smooth functions is the usual derivative of these functions.

2) In our approach, using GFD we may define the derivative of continuous nonsmooth functions which the other approaches are defined usually for special functions such as Lipschiptz or convex functions.

3) Our approach for obtaining GFD is a global approach, in which the other methods and definitions are applied for one fixed known point. 
4) Calculating GFD by our approach is easier than other available approaches.

\section{References}

[1] F. H. Clarke, "Optimization and Non-Smooth Analysis," Wiley, New York, 1983.

[2] V. F. Demyanov and A. M. Rubinov, "Constructive Nonsmooth Analysis," Verlag Peter Lang, New York, 1995.

[3] W. Schirotzek, "Nonsmooth Analysis," Springer, New York, 2007. doi:10.1007/978-3-540-71333-3

[4] B. Mordukhovich, "Approximation Methods in Problems of Optimization and Control," Nauka, Moscow, 1988.

[5] B. Mordukhovich, "Complete Characterizations of Openness, Metric Regularity, and Lipschitzian Properties of Multifunctions," Transactions of the American Mathematical Society, Vol. 340, 1993, pp. 1-35. doi: $10.2307 / 2154544$

[6] B. S. Mordukhovich, "Generalized Differential Calculus for Nonsmooth and Set-Valued Mappings," Journal of Mathematical Analysis and Applications, Vol. 183, No. 1, 1994, pp. 250-288. doi:10.1006/jmaa.1994.1144

[7] B. Mordukhovich, "Variational Analysis and Generalized Differentiation," Vol. 1-2, Springer, New York, 2006.

[8] B. Mordukhovich, J. S. Treiman and Q. J. Zhu, "An Extended Extremal Principle with Applications to Multiobjective Optimization," SIAM Journal on Optimization, Vol. 14, 2003, pp. 359-379. doi:10.1137/S1052623402414701

[9] A. D. Ioffe, "Nonsmooth Analysis: Differential Calculus of Nondifferentiable Mapping," Transactions of the American Mathematical Society, Vol. 266, 1981, pp. 1-56. doi:10.1090/S0002-9947-1981-0613784-7

[10] A. D. Ioffe, “Approximate Subdifferentials and Applica- tions I: The Finite Dimensional Theory," Transactions of the American Mathematical Society, Vol. 281, 1984, pp. 389-416.

[11] A. D. Ioffe, "On the Local Surjection Property," Nonlinear Analysis, Vol. 11, 1987, pp. 565-592. doi:10.1016/0362-546X(87)90073-3

[12] A. D. Ioffe, "A Lagrange Multiplier Rule with Small Convex-Valued Subdifferentials Fornonsmooth Problems of Mathematical Programming Involving Equality and Nonfunctional Constraints," Mathematical Programming, Vol. 588, 1993, pp. 137-145. doi:10.1007/BF01581262

[13] A. D. Ioffe, "Metric Regularity and Subdifferential Calculus," Russian Mathematical Surveys, Vol. 55, No. 3, 2000, pp. 501-558. doi:10.1070/RM2000v055n03ABEH000292

[14] M. S. Gowda and G. Ravindran, "Algebraic Univalence Theorems for Nonsmooth Functions," Journal of Mathematical Analysis and Applications, Vol. 252, No. 2, 2000, pp. 917-935. doi:10.1006/jmaa.2000.7171

[15] R. T. Rockafellar and R. J. Wets, "Variational Analysis," Springer, New York, 1997.

[16] P. Michel and J.-P. Penot, "Calcul Sous-Diff"erentiel Pour des Fonctions Lipschitziennes et Non-Lipschitziennes," CR Academic Science Paris, Ser. I Math. Vol. 298, 1985, pp. 269-272.

[17] J. S. Treiman, "Lagrange Multipliers for Nonconvex Generalized Gradients with Equality, Inequality and Set Constraints," SIAM Journal on Optimization, Vol. 37, 1999, pp. 1313-1329. doi:10.1137/S0363012996306595

[18] E. Stade, "Fourier Analysis, USA," Wiley, New York, 2005.

[19] M. S. Bazaraa, J. J. Javis and H. D. Sheralli, "Linear Programming," Wiley \& Sons, New York, 1990.

[20] M. S. Bazaraa, H. D. Sheralli and C. M. Shetty, "Nonlinear Programming: Theory and Application," Wiley \& Sons, New York, 2006. 